Andressa F Ribeiro'

Gisela R A M Marques

Júlio C Voltolini"

Maria Lúcia F Condino'

\title{
Associação entre incidência de dengue e variáveis climáticas
}

\author{
Association between dengue \\ incidence and climatic factors
}

\section{RESU MO}

OBJETIVO: Descrever a ocorrência de casos de dengue autóctone segundo sexo, faixa etária e local provável de infecção e sua relação com variáveis climatológicas.

MÉTODOS: Os registros de casos autóctones em São Sebastião, SP, de 2001 a 2002, e confirmados laboratorialmente foram estudados. A densidade larval foi verificada pelos índices de predial, recipientes e Breteau. A relação entre dados de pluviosidade, temperatura e número de casos foi analisada pela correlação de Spearman utilizando-se o conceito time-lag.

RESULTADOS: Os coeficientes de incidência anuais para 2001 e 2002 foram de 80,31 e 211,1 por 10.000 habitantes, respectivamente. A maioria dos casos de dengue $(\mathrm{n}=1.091 ; 65 \%)$ foi registrada na área central do município. O sexo feminino foi o mais acometido $(n=969 ; 60 \%)$ e ambos os sexos nas faixas etárias entre 20 e 29 e 30 e 39 anos. Não foi observada correlação entre variáveis climatológicas e número de casos do mesmo mês, entretanto, esta associação ocorre a partir do segundo mês estendendo-se até o quarto mês.

CONCLUSÕES: A associação entre o número de casos de dengue e fatores abióticos identificou o intervalo de tempo em que a chuva e a temperatura contribuíram na geração de novos casos. Tais aspectos, associados à vulnerabilidade turística da região litorânea, propiciaram condições para ocorrência da doença. A urbanização sem a devida estrutura de saneamento possivelmente influenciou na densidade de mosquitos e na incidência de dengue. Esses fatores podem ter contribuído para a dispersão do mosquito e disseminação dos vários sorotipos da doença.

DESCRITO RES: Aedes aegypti. Dengue, epidemiologia. Fatores abióticos. Epidemiologia descritiva.

\section{ABSTRACT}

Superintendência de Controle de

" Departamento de Ciências Biológicas. Universidade de Taubaté. Taubaté, SP, Brasil

Correspondência | Correspondence: Andressa Francisca Ribeiro Laboratório de Culicídeos da Sucen - SR-3 Taubaté

Praça Coronel Vitoriano, 23

12020-020, Taubaté, SP, Brasi

E-mail: labpesqsr3@sucen.sp.gov.br

Recebido: 15/7/2005 Revisado: 23/2/2006 Aprovado: $2 / 3 / 2006$ 
29 and 30 to 39 years old of both sexes were mostly affected. Significant associations were not observed between climatic variables and the number of dengue cases in the first month; however, this association became evident in the second month throughout the fourth month.

CONCLUSIONS: The association between the number of dengue cases and abiotic factors identified the time lag in which rain and temperature favored the occurrence of new cases. These aspects, associated to tourist vulnerability in the coastal area, promoted conditions for dengue occurrence. The urbanization without sanitary infrastructure possibly affected mosquito density and dengue incidence. Such factors may have contributed to mosquito dispersion and dissemination of different dengue serotypes.

KEYW O RDS: Aedes aegypti. Dengue, epidemiology. Abiotic factors. Epidemiology, descriptive.

\section{INTRO DUÇÃO}

A dengue é uma arbovirose que tem causado preocupação por ser um problema de saúde pública mundial. Os países tropicais são os mais atingidos em função de suas características ambientais, climáticas e sociais. Essa doença caracteriza-se por ser febril aguda, cujo agente etiológico é constituído por quatro sorotipos: DEN-1, 2, 3 e 4. A transmissão ocorre principalmente pela picada de mosquitos Aedes aegypti infectados, os quais possuem hábito domiciliar. Sua convivência com o homem é favorecida pela utilização de recipientes artificiais no desenvolvimento das formas imaturas, condição ecológica que torna esta espécie predominantemente urbana. ${ }^{3}$

A expansão das áreas de ocorrência de dengue no mundo e no Brasil está associada tanto à urbanização, sem a devida estrutura de saneamento, quanto à "globalização" da economia. Tais fatores contribuem não só para a dispersão ativa do mosquito como também para a disseminação dos vários sorotipos da doença. A transmissão no Estado de São Paulo teve início em 1987 e desde então vem apresentando tendência de crescimento/ascensão: em 2001 foram confirmados 51.472 casos configurando-se como a maior incidência de dengue já observada. ${ }^{13}$

A dengue tem acometido indivíduos de ambos os sexos, porém existem estudos que mostram maior incidência em mulheres do que em homens. Pessoas de todas as idades são suscetíveis de adquirir esta infecção, contudo a maior incidência de casos nas faixas etárias mais elevadas é um padrão observado em áreas indenes logo após a introdução de um sorotipo do vírus. ${ }^{5}$

A incidência de casos de dengue também flutua com as condições climáticas e está associada com o aumento da temperatura, pluviosidade e umidade do ar, condições que favorecem o aumento do número de criadouros disponíveis e também o desenvolvimento do vetor. ${ }^{1}$

Moore ${ }^{8}$ (1985), analisando a abundância de Aedes aegypti em relação a dados climáticos, mostrou que a temperatura não foi um bom indicador de abundância larval, porém o volume e o número de dias com chuva podem constituir preditores úteis de sua abundância. Segundo Reiter ${ }^{10}$ (1988) isso geralmente é aceito, entretanto, deve-se considerar que as taxas de infecção viral no vetor também variam com as condições climáticas.

O presente estudo teve por objetivo descrever a ocorrência de casos de dengue autóctone segundo sexo, faixa etária e local provável de infecção e sua relação com variáveis climatológicas, visando ao aprimoramento da vigilância e controle da doença no município.

\section{MÉTO DOS}

O estudo foi realizado em São Sebastião, localizado nas proximidades de centros urbanos maiores, anteriormente infestados. Trata-se de cidade balneária com temperatura média anual e pluviosidade elevadas, respectivamente de $22,5^{\circ} \mathrm{C}$ e ao redor de $1.500 \mathrm{~mm} /$ ano, enquadrando-se no clima tropical úmido.

Localizado no litoral norte do Estado de São Paulo, o município de São Sebastião situa-se entre as coordenadas latitude $23^{\circ} 21^{\prime} 20^{\prime \prime} \mathrm{S}$ e longitude $45^{\circ} 21^{\prime} 00^{\prime \prime} \mathrm{W}$, a $220 \mathrm{~km}$ da capital paulista. Sua superfície territorial é de $520 \mathrm{~km}^{2}$, sendo aproximadamente $300 \mathrm{~km}^{2}$ cobertos por Mata Atlântica. Segundo o censo demográfico do ano de 2000, a população total era de 58.038 habitantes. ${ }^{6}$ Atualmente sua área urbana extrapola os limites das planícies invadindo as montanhas costeiras. Este processo intenso e desordenado de ocupação, pres- 
sionado pelo crescimento da população urbana e flutuante colabora para o agravo dos serviços de saneamento básico.

Em 1996, o município teve seu primeiro registro da presença de Aedes aegypti. ${ }^{9}$ Embora alvo sistemático de atividades de vigilância, o vetor encontrou condições favoráveis ao seu desenvolvimento ocasionando infestação domiciliar em 2000 e registros de casos autóctones da doença a partir de 2001.

Para determinar o número de casos, distribuição por localidade, sexo, faixa etária e sorotipo circulante, foi analisada a totalidade dos casos positivos autóctones confirmados laboratorialmente e registrados no Sistema de Vigilância Epidemiológica (SINAN - Sistema de Informação de Agravos de Notificação) para 2001 e 2002. Para testar a proporção de casos em homens e mulheres foi utilizado o teste para comparação entre duas proporções independentes.

Para expressar a infestação larvária por ocasião do estudo, foram utilizados três índices: índice predial, índice de recipientes e índice de Breteau.*

O estudo é do tipo descritivo com delineamento ecológico, utilizando o conceito time lag. Esta definição é utilizada por Depradine \& Lovell $^{1}$ (2004) e permite investigar os fenômenos resultantes das interações com o ambiente num determinado espaço de tempo. Para esses autores, o clássico procedimento de correlação simultânea para identificar relações entre casos de dengue e variáveis climáticas fornece correlações fracas de maneira geral, embora estatisticamente significantes. No referido estudo é evidenciado um intervalo de tempo para a ocorrência de associações mais explícitas. Fatores como: período de desenvolvimento embrionário, tempo de eclosão de larvas, tempo de desenvolvimento das formas imaturas e período de incubação (extrínseco e intrínseco) constituem-se componentes que contribuem para explicar a necessidade de considerar o intervalo de tempo (time lag) na busca de associações entre tais eventos.

Os dados de pluviosidade e de temperatura média mensal foram obtidos no Instituto Agronômico de Campinas, Estação Experimental de Ubatuba. A correlação de Spearman foi utilizada para analisar a relação entre pluviosidade, temperatura e número de casos. Os dados foram analisados utilizando-se o programa Statistica 5.05.

\section{RESU LTADO $S$}

Foram registradas 3.442 notificações de casos suspeitos de dengue, sendo $1.282 \mathrm{em} 2001$ e $2.152 \mathrm{em}$ 2002. No primeiro ano de epidemia no município, foram confirmados 466 casos autóctones, representando 36\% das notificações; em 2002, esse número subiu para 1.225 casos positivos, correspondendo a $57 \%$ das notificações registradas.

As ondas epidêmicas registradas em São Sebastião, concentraram-se de abril a junho. Nos anos de $2001 \mathrm{e}$ 2002, as respectivas taxas anuais de incidência foram 80,3 e 211,1 por 10.000 habitantes. No ano de 2001 a referida taxa apresentou no mês de junho o seu valor máximo de 33,7 casos por 10.000 habitantes. Em 2002, o mês de abril apresentou a maior taxa, 93,1 casos de dengue por 10.000 habitantes sendo este o mais alto coeficiente de incidência registrado durante o estudo. Verificou-se redução dos valores nos meses subseqüentes, com o desaparecimento de casos positivos no último trimestre dos anos de 2001 e 2002. As maiores incidências de dengue coincidiram com a estação do outono: 1.163 casos, que corresponderam a $70 \%$ do total registrado nos dois anos (Figura).

Com relação à circulação de sorotipos da dengue em 2001, foram realizados 36 isolamentos virais, sendo um exame positivo para DEN-2 e 35 para o DEN-1.

A distribuição de casos segundo o local provável de infecção indica que a dengue ocorreu de maneira diferenciada nas várias localidades do município estudado. As áreas de ocorrência da doença foram coincidentes com as de residência. Das 41 localidades com transmissão, somente os bairros centrais (Centro, Topolândia, e Itatinga) se destacaram em relação aos demais, apresentando 1.091 casos positivos (65\%), 
Tabela 1 - Índice de Breteau, Infestação Predial e Índice de Recipientes (Aedes aegypti). São Sebastião, SP, 2001-2002.

\begin{tabular}{|c|c|c|c|c|c|c|}
\hline \multirow{2}{*}{$\begin{array}{l}\text { Ano } \\
\text { M ês }\end{array}$} & \multicolumn{3}{|c|}{2001} & \multicolumn{3}{|c|}{2002} \\
\hline & IB & IP & IR & IB & IP & IR \\
\hline Janeiro & - & - & - & 29 & 12,2 & 6 \\
\hline Fevereiro & - & - & - & 16,7 & 9,1 & 8,8 \\
\hline Março & - & - & - & 3,3 & 3,1 & 3,3 \\
\hline Abril & - & - & - & 7,4 & 4,9 & 4,2 \\
\hline Maio & - & - & - & - & - & - \\
\hline Junho & - & - & - & 5,1 & 4 & 3 \\
\hline Julho & 2,7 & 2,4 & 2 & 4,2 & 3,2 & 1,4 \\
\hline Aqosto & 1,2 & 1,2 & 0.8 & 5,6 & 4,7 & 3 \\
\hline Setembro & 11,3 & 7,8 & 3,1 & 8,5 & 6 & 2,5 \\
\hline O utubro & - & - & - & 5 & 4,2 & 1,1 \\
\hline Novembro & 6,37 & 4,9 & 2,9 & 13,2 & 8,3 & 3,6 \\
\hline D ezembro & 10,8 & 8,33 & 5,54 & 11,9 & 7,8 & 2,4 \\
\hline
\end{tabular}

IB: Índice de Breteau; IP: Índice Predial; IR: Índice de Recipiente

Fonte: Sucen Taubaté Serviço Regional-03

reforçando o aspecto urbano da doença, pois esses bairros concentram a maior parte da população residente.

Constatou-se que aproximadamente $60 \%$ dos casos ocorreram no sexo feminino $(n=969)$ e $40 \%(n=721)$ no masculino. Com relação à distribuição da doença por grupos etários, observou-se a ocorrência de casos em todos os intervalos, com concentração nas idades entre 20 a 29 anos e 30 a 39 anos, em ambos os sexos (Tabela 1).

Os valores dos índices de infestação predial, recipiente e de Breteau para Aedes aegypti variaram no período de estudo, revelando elevadas densidades nos primeiros e últimos meses do ano (Tabela 2). Os maiores valores de temperatura e umidade relativa do ar foram registrados entre novembro a abril, aproximadamente os mesmos meses onde foram observados os maiores valores de densidade larvária de Aedes aegypti.

Os resultados de correlação entre casos de dengue e variáveis climáticas encontram-se na Tabela 3 . Não foi observada associação entre os valores de tempe- ratura média mensal, pluviosidade média mensal e número de casos do mesmo mês de análise. A correlação passa a ser observada ao serem considerados os valores dos fatores abióticos de um determinado mês com o número de casos do mês seguinte.

\section{DISCUSSÃO}

Observou-se que os valores dos índices de infestação predial, recipiente e de Breteau para Aedes aegypti variaram no período, revelando elevadas densidades nos primeiros e últimos meses do ano (Tabela 1). Esses índices são utilizados por muitos países para monitorar os níveis de infestação, porém, nem sempre apresentam correlação com a incidência de dengue, que eventualmente é registrada na vigência de índices de Breteau baixos. ${ }^{2}$ No entanto, os índices de infestação vetorial apontaram elevada densidade larvária, o que pode ter propiciado maior número de picadas e, conseqüentemente, maior risco de transmissão.

Segundo Passos et $\mathrm{al}^{9}$ (2003), o estabelecimento da infestação domiciliar pelo Aedes aegypti em São Se-

Tabela 2 - Taxa de incidência de dengue segundo sexo e faixa etária. São Sebastião, SP, 2001-2002.

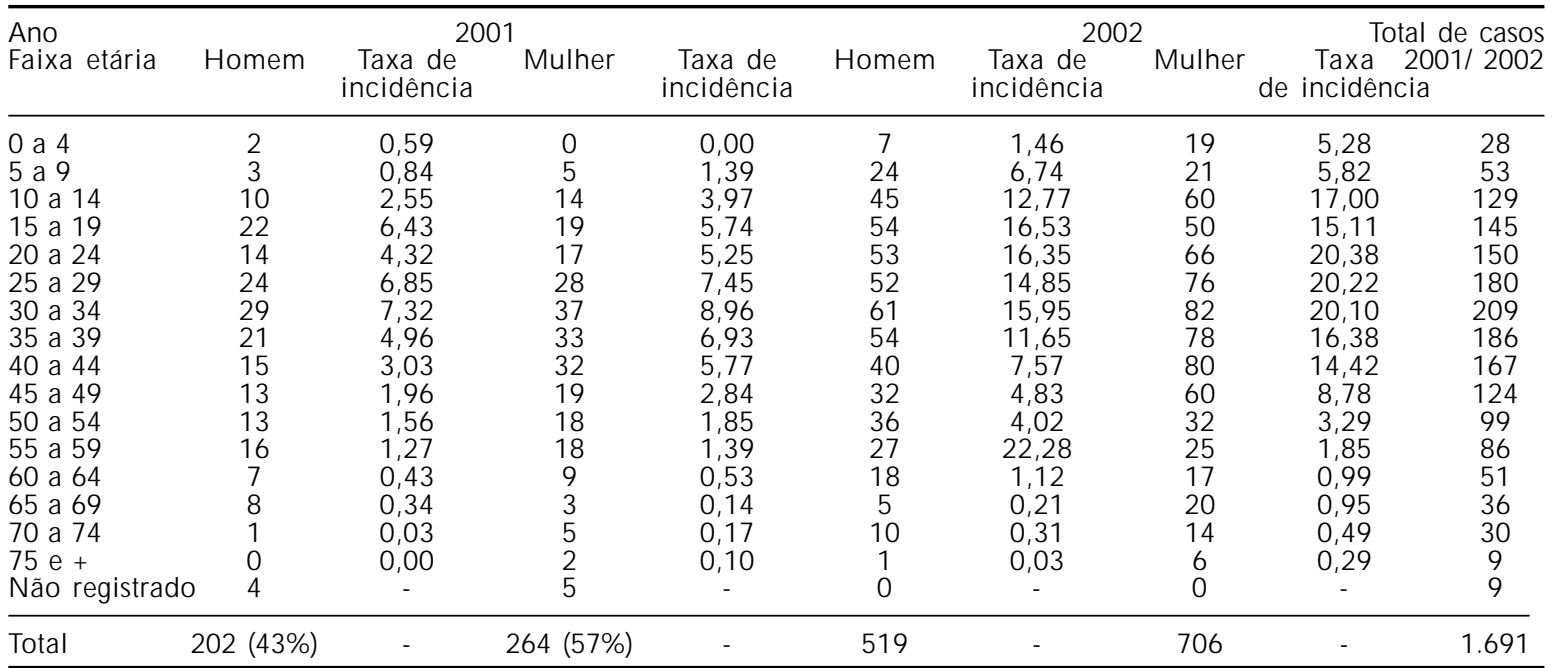

Fonte: Instituto Brasileiro de Geografia e Estatística e Sistema de Informações de Agravos de Notificação 
Tabela 3 - Associação entre o número de casos e fatores abióticos considerando time lag. São Sebastião, SP, 2001-2002.

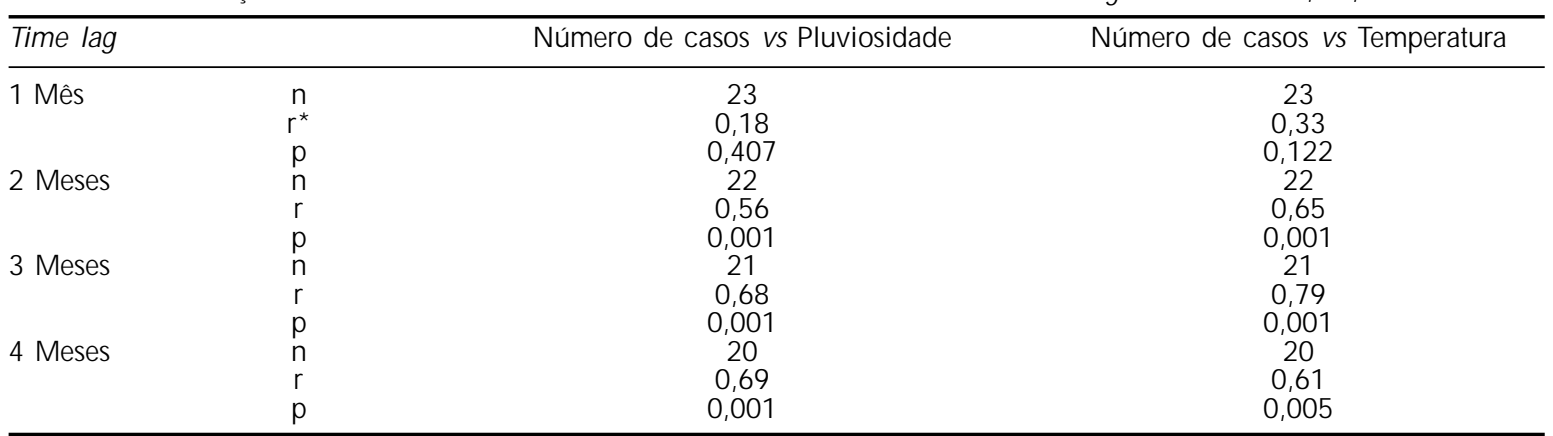

*Correlação de Spearman

bastião ocorreu no final de 2000. O processo epidêmico teve início em fevereiro de 2001, indicando assim um curto espaço de tempo entre infestação domiciliar e início de epidemia.

Os resultados da distribuição da doença segundo sexo são semelhantes aos de Vasconcelos et $\mathrm{al}^{13}$ (1993). Esses autores comentam que uma das explicações para esta diferença entre os sexos seria a maior permanência da mulher no intradomicílio ou peridomicílio, locais onde predominantemente ocorre a transmissão de dengue. Porém, os dados do presente estudo discordam com os obtidos por Gonçalves Neto \& Rebêlo $^{5}$ (2004), onde foi encontrada transmissão similar entre os sexos.

Corroborando com os resultados de Gòmez-Dantés et $\mathrm{al}^{4}$ (1995) no México, São Sebastião apresentou taxas de incidências que aumentaram com a idade, até o intervalo entre 30 a 39 anos, decrescendo sensivelmente a partir de então.

Segundo Keating ${ }^{7}$ (2001), entre outros fatores, a temperatura e a pluviosidade afetam a sobrevivência, a reprodução do vetor, as mudanças na sua distribuição e a densidade. Esses fatores abióticos climáticos têm mostrado associação com casos de dengue. $\mathrm{O}$ padrão sazonal de incidência da doença coincide com o verão, devido a maior ocorrência de chuva e aumento de temperatura nesta estação. ${ }^{8,12}$

De acordo com Gonçalves Neto \& Rebêlo ${ }^{5}$ (2004) as chuvas exercem grande influência na determinação do período de ocorrência da doença. Nesse estudo, a pluviosidade não só aumentou consideravelmente a quantidade de criadouros disponíveis para o desenvolvimento das formas imaturas do vetor, como também gerou condições ambientais mais apropriadas para o desenvolvimento de adultos.

A relação entre número de casos de dengue e fatores abióticos tem sido relatada por diversos autores. ${ }^{1,7,8}$ No presente estudo foi observada a associação entre o número de casos, pluviosidade e temperatura considerando o tempo entre o fato biológico, a transmissão, e o registro dos casos no sistema de informação. Esse time-lag revelou associação significativa no segundo, terceiro e quarto mês de observação, ou seja, a chuva e a temperatura de um determinado mês contribuíram para explicar o número de casos de dengue de dois até quatro meses depois. As respectivas forças de associação aumentaram e depois se estabilizaram, variando de 60 a $80 \%$ dependendo do mês no referido período.

Embora tenha sido evidenciado um contexto ecológico regional, a dinâmica de transmissão de dengue envolve outros fatores determinantes. Assim, deve ser considerada a limitação dos presentes resultados.

De acordo com Forattini ${ }^{3}$ (2002), as condições climáticas, caracterizadas pelas precipitações atmosféricas e temperaturas elevadas, em geral mostram relação positiva com a transmissão de dengue. O conhecimento desse processo poderá propiciar maior entendimento sobre a dinâmica de transmissão e, conseqüentemente, contribuir para o seu controle.

Em relação aos coeficientes de infecção, sugere-se que os coeficientes mensais e anuais podem ser mais elevados do que os registrados no presente estudo. Gómez-Dantés et $\mathrm{al}^{4}$ (1995) estimaram que os casos identificados pela vigilância epidemiológica mexicana corresponderam a apenas $10 \%$ do total de casos positivos, apontando elevada subnotificação. Scandar et $\mathrm{al}^{11}$ (1998) identificaram a relação de um caso notificado para aproximadamente 12 habitantes infectados não notificados em São José do Rio Preto.

A circulação viral no município provavelmente deve ter se estabelecido nos primeiros meses do ano e a inexistência de imunidade da população propiciaram sua explosão. Em conclusão, os isolamentos virais registraram a circulação de dois sorotipos diferentes, indicando possibilidade da ocorrência de febre hemorrágica da dengue. Os dados obtidos reforçam tra- 
tar-se de doença urbana, pois a transmissão ocorreu em sua maior parte na região central do município, coincidindo com a área de residência dos casos confirmados, considerada área superpovoada e com elevados níveis de infestação do vetor.

Os resultados encontrados mostraram a existência de associação entre número de casos de dengue e fatores abióticos, identificando o intervalo de tempo em que a

\section{REFERÊNCIAS}

1. Depradine CA, Lovell EH. Climatological variables and the incidence of dengue fever in Barbados. Int J Environ Health Res. 2004;14:429-41.

2. Donalísio $M R$, Glasser $C M$. Vigilância entomológica e controle de vetores do dengue. Rev Bras Epidemiol. 2002;5:259-72.

3. Forattini OP. Culicidologia médica: identificação, biologia e epidemiologia. São Paulo: Edusp; 2002. v. 2.

4. Gómez Dantes H, Ramos Bonifaz B, Tapia Conyer R. El riesgo de transmisión de dengue: um espacio para la estratificación. Salud Pública Méx. 1995;37(Supl):88-97.

5. Gonçalves Neto VS, Rebêlo JMM. Aspectos epidemiológicos do dengue no município de São Luis, Maranhão, Brasil, 1997-2002. Cad Saúde Pública. 2004;20:1424-31.

6. Instituto Brasileiro de Geografia e Estatística - IBGE. Censo demográfico, 2000. Rio de Janeiro; 2000.

7. Keating J. An investigation into the cyclical incidence of dengue fever. Soc Sci Med. 2001;53:1587-97. chuva e a temperatura contribuíram na geração de novos casos. Tais aspectos podem ser úteis para elaboração de estratégias de controle e/ou planejamento, especialmente em cidades com perfil semelhante. $\mathrm{O}$ aumento de densidade populacional no referido município coincide com os meses de verão, com os maiores valores de temperatura e pluviosidade, e conseqüientemente, elevados índices vetoriais. Esse cenário reúne as condições necessárias para ocorrência de dengue.

8. Moore CG. Predicting Aedes aegypti abundance from climatological data. In: Lounibos LP, Rey JR, Frank JH, editors. Ecology of mosquitoes. Vero Beach (FL): Florida Medical Entomology Laboratory; 1985. p. 223-33.

9. Passos RA, Marques GRAM, Condino LMF, Voltolini JC. Dominância de Aedes aegypti sobre Aedes albopictus no litoral sudeste do Brasil. Rev Saúde Pública. 2003;37:729-34.

10. Reiter P. Weather, vector biology and arboviral recrudescense. In: Monath TP, editor. The arboviruses: epidemiology and ecology. Boca Raton (FL): CRC Press; 1988. p. 245-55.

11. Scandar SAS, Cardoso Júnior RP, Goldenberg $P$, Ferreira IB, Souza LTM. Inquérito sorológico, após epidemia de dengue: Paraíso, São Paulo. Rev Inst Adolfo Lutz. 2003;62:83-9.

12. Teixeira MG, Costa MCN, Barreto LM, Barreto FR Epidemiologia da dengue em Salvador - Bahia, 19951999. Rev Soc Bras Med Trop. 2001;34:269-74.

13. Vasconcelos PFC. Epidemia de febre clássica de dengue causada pelo sorotipo 2 em Araguaína, Tocantins, Brasil. Rev Inst Med Trop São Paulo. 1993;35:141-8. 